(pp 404-409)

\title{
Effects of Mental Stress on the Pain-related SEP
}

\author{
Yoichi SATO, PT \\ Department of Rehabilitation, Nasu Red Cross Hospital \\ Hideaki ONISHI, PT, PhD \\ Department of Physical Therapy, Niigata University of Health and Welfare
}

Purpose: The objective of this study was to determine whether psychological stress is associated with changes in subjective pain intensity and changes in excitability of the somatosensory cortex.

Methods: Twenty healthy volunteers participated in this study. Of these, 10 took the Uchida-Kraeplin psychodiagnostic test as a mental stress task, whereas the remaining 10 (control group) were seated comfortably for approximately 35 minutes. Painful electrical stimuli were applied to the right median nerve of participants in both groups. We compared pain-related somatosensory evoked potentials after painful electrical stimulation between the test and control groups and pain intensity was rated according to the visual analog scale (VAS) during electrical stimulation before and after the mental stress task in the test group.

Results: VAS scores for pain intensity and the peak-to-peak amplitude of N20/P25 were found to be significantly increased after the mental stress task.

Conclusion: We concluded that mental stress increased subjective pain intensity and excitability in the primary somatosensory cortex. 